\title{
Paarbildung und Paarzusammenhalt bei der monogamen Wüstenassel Hemilepistus reaumuri (Crustacea, Isopoda, Oniscoidea)
}

\author{
Von K. Eduard und Christa LinsenMair
}

Mit 6 Abbildungen

Eingegangen am 6.2. 1971

Inhalt: A. Einleitung, Vorversuche und Problemstellung S. 134. - B. Beobachtungsgebiete und -zeit S. 125. - C. Ergebnisse: I. Vergleich der Situation im Sommer und Winter mit der im Frühjahr S. 135. - II. Paarbildung S. 135. - 1. Höhlenerwerb S. 136. - 2. Das Verhalten des einzelstehenden Höhlenbesitzers S. 136. - 3. Erkennen und Aufsuchen des Geschlechtspartners S. 136. - III. Paarzusammenhalt S. 141. - 1. Das individuelle Erkennen des Partners S. 142. Das Sich-kennenlernen der Partner S. 146. - 3. Das Verhalten der verpaarten Asseln zueinander S. 147. - 4. Das Verhalten nach Verlust des Partners S. 148. - D. Diskussion S. 151. - Zusammenfassung S. 153. - Summary S. 154. - Literaturverzeichnis S. 155.

Der Deutschen Forschungsgemeinschaft danken wir für die finanzielle Unterstützung.

\section{A. Einleitung, Vorversuche und Problemstellung}

In den festen Sandböden mancher nordafrikanischer Halbwüstengebiete fallen zahlreiche Höhlen mit etwa $1 \mathrm{~cm}$ Eingangsdurchmesser auf. Während der heißen Jahreszeit erscheinen ihre Bewohner - Wüstenasseln der Art Hemilepistus reaumuri - mit oder kurz nach Sonnenuntergang zur Nahrungssuche an der Oberfläche. Zwei bis vier Stunden nach Sonnenaufgang verschwinden sie wieder. Im Frühjahr kann man sie während des gesamten Tages außerhalb der Höhlen antreffen. Wüstenasseln zählen in ihrem Lebensraum zu den häufigsten Tieren: an dicht besiedelten Stellen leben im Spätsommer auf $100 \mathrm{qm}$ bis weit über 10000 Individuen. Die Tatsache, daß es Asseln mit offensichtlich großem Erfolg gelungen ist, in derartig lebensfeindliche Biotope einzudringen, verdient Beachtung.

Setzt man Wüstenasseln während der heißen Stunden eines Sommertags auf die besonnte Bodenoberfläche (etwa $50-60^{\circ} \mathrm{C}$ ), dann sterben sie schon nach etwa 15-30 Minuten. Überleben kann nur die Wüstenassel, die zu bestimmten Zeiten Unterschlupf in einer Höhle findet. Man muß daher erwarten, daß die Asseln über Verhaltensmechanismen verfügen, die ihnen rechtzeitigen Zugang und sicheren Platz in einer Höble garantieren.

Im Sommer ziehen sich die Asseln nach ihren Ausflügen immer in schon vorhandene Höhlen zurück. Gräbt man die zwischen 1-2 $\mathrm{m}$ langen und $0,4-0,8 \mathrm{~m}$ tiefen Höhlen auf, dann kann man über 60 Individuen in einem Bau finden. Handelt es sich hierbei um eine zufällige Aggregation, die sich von Aktivitätsperiode zu Aktivitätsperiode ändert? Markiert man die Bewohner einer Höhle und beobachtet sie über mehrere Tage, dann fallen 3 bemerkenswerte Tatsachen auf: 
1. Die Insassen einer Höble kehren nach ibren Ausflügen regelmäßig zu dieser Höble zurück. 2. Es bandelt sich um eine geschlossene Gemeinschaft. Ibre Mitglieder erkennen sich gegenseitig und verteidigen ibre Höble gegen Fremde. 3. Innerhalb der Gemeinschaft findet man Zusammenarbeit und Arbeitsteilung.

Ziel der vorliegenden und nachfolgender Untersuchungen ist es, Entstehung, Entwicklung und ökologischen Anpassungswert der Gemeinschaften - vor allem im Hinblick auf Probleme ethologischer Adaptationen an ein Extrembiotop - zu analysieren.

Über Verhalten und Ökologie von $H$. reaumuri liegen nur bruchstückhafte Einzelbeobachtungen vor (VANDEL 1924, VERrier 1932, Cloudsley-Thompson 1956a, Edney 1958, Warburg 1968). Marikovsky (1969) beobachtete eine verwandte Art, H. rhinoceros, am Balkasch-See.

\section{B. Beobachtungsgebiete und -zeit}

Unser Hauptbeobachtungsgebiet lag in Südtunesien, etwa $15 \mathrm{~km} \mathrm{SSE} \mathrm{von} \mathrm{Gabes,} \mathrm{etwa} 4 \mathrm{~km}$ von der Küste entfernt und zwischen 5-20 m über Meereshöhe. Es gehört zur geographischen Litoralzone des Golfes von Gabes, was eine Milderung des saharischen Klimas zur Folge hat: mittlere Temperatur im kältesten Monat (Januar) $10,8^{\circ} \mathrm{C}$, im heißesten Monat (August) $27,4^{\circ} \mathrm{C}$; absolutes Minimum $-3^{\circ} \mathrm{C}$, absolutes Maximum $53^{\circ} \mathrm{C}$ (die metereologischen Daten wurden in den Jahren 1901 -1960 in Gabes gesammelt, s. Mensching 1968). Niederschlagmäßig zählt diese Region zu den ariden Gebieten Tunesiens $(<200 \mathrm{~mm}$ im Jahresmittel, in Trockenjahren $<100 \mathrm{~mm})$. Die steppenmäßige Vegetation setzt sich aus einer artenreichen einjährigen Kräuterflora, die größtenteils bereits im Mai verdorrt ist, und perennierenden, durch starke Beweidung meist verkrüppelten Büschen und Sträuchern zusammen. Der Boden besteht überwiegend aus Feinsand, der nach Durchfeuchtung und anschließender Trocknung sehr hart wird. Flugsand - ein, die Verbreitung von $H$. reaumuri limitierender Faktor - findet sich in nennenswerter Menge nur in den das Beobachtungsgebiet durchziehenden Trockentälern.

1969 verbrachten wir das Ende des Monats August und die erste Septemberhälfte in diesem Gebiet, 1970 hielten wir uns von Anfang März bis Anfang Mai und im Dezember (und Anfang Januar 1971) dort auf.

$\mathrm{Zu}$ kürzeren Beobachtungen hatten wir in den Jahten 1963 in Tunesien und Ägypten, wo H. reaumuri auch in echte Wüstengebiete vorgedrungen ist, und 1968 in Libyen Gelegenheit.

\section{Ergebnisse}

\section{Vergleich der Situation im Sommer und Winter mit der im Frühjahr}

Im Sommer und Winter beherbergten mit wenigen Ausnahmen $(<5 \%)$ alle bewohnten Höhlen Gruppen von wenigstens 10 bis 62 Individuen. In der 2. Märzwoche dagegen fanden wir in 70 von 100 Höhlen ein Pärchen; in den restlichen 30 hielt sich nur ein o oder ein $0 *$ auf.

Nach diesen Beobachtungen sind folgende Annahmen naheliegend und müssen überprüft werden:

1. Gründer der Höhlengemeinschaft ist ein Pärchen. 2. Die Höhlengemeinschaft ist ein Familienverband. 3. Die Höhlengemeinschaften lösen sich zu Beginn des Frühjahrs auf.

Die folgenden Ausführungen beschäftigen sich mit dem 1. Punkt.

\section{Paarbildung}

Begegnen sich zwei gleich- oder verschiedengeschlechtliche Wüstenasseln außerhalb einer Höhle oder deren nächster Umgebung, dann reagieren sie entweder überhaupt nicht aufeinander oder sie weichen sich - vor allem nach Berührung - aus. Nie fanden wir Anhaltspunkte, die auf eine Paarbildung außerhalb der Höhle schließen ließen. 


\section{Höhlenerwerb}

Von Anfang März bis Anfang Mai konnten wir in einem Gebiet, in dem mehr als 10000 Asseln lebten, nur 18 우 und 10 o $^{\star} o^{\star}$ direkt beim Neubau einer Höhle beobachten. Asseln ohne eigene Höhle versuchten im Frühjahr grundsätzlich, in eine schon bewohnte Höhle einzudringen (s. S. 137) oder eine leere - meist vorjährige - Höhle in Besitz zu nehmen. Letzteres taten $\sigma^{*} \sigma^{*}$ und of mit der gleichen Bereitschaft, wie sehr zahlreiche Beobachtungen und folgendes Experiment zeigten: in einem $3 \mathrm{~m} \times 5 \mathrm{~m}$ messenden Areal des Untersuchungsgebietes wurden aus 15 Höhlen die oder der Besitzer entfernt. Mehrmals täglich wurde kontrolliert, ob und von wem diese Höhlen besetzt wurden. Neue Insassen entfernten wir gleich wieder. Innerhalb von 7 Tagen hatten insgesamt 14 Asseln, und zwar $8 \delta^{\lambda} \delta^{A}$ und 6 oq - jeweils allein - eine der freien Höhlen bezogen.

\section{Das Vethalten der einzelstehenden Höhlenbesitzer}

Einzelstehende Höhleneigentümer sitzen - immer Kopf dem Eingang zugewandt - während der Aktivitätsphasen ${ }^{1}$ ) normalerweise am Eingang (s. Abb. 1) oder nur $1-3 \mathrm{~cm}$ darunter. Bei ungünstigen Witterungsbedingungen (unter $14^{\circ} \mathrm{C}$, über $35^{\circ} \mathrm{C}$, starkem Wind, leichtem Regen) und in der Zeit zwischen den Aktivitätsphasen sitzen sie meist tiefer in ihren Höhlen. Ist die neuerworbene Höhle länger als $4-5 \mathrm{~cm}$, dann baut der Besitzer nur selten. Nur 3 von 10 Asseln verlassen während der ersten 24 Std. nach Inbesitznahme ihre Höhlen zur Nahrungssuche. Diese Ausflüge, die bis etwa $1 \mathrm{~m}$ vom Höhleneingang wegführen, dauerten in den beobachteten 8 Fällen nicht länger als 1/2 Std., im Mittel rund 15 Min. Die Besitzer kehren danach immer zu ihren Höhlen zurück. 4 von 10 einzelstehenden Höhlenbesitzern entfernen sich sogar innerhalb von $72 \mathrm{Std}$. nicht von ihrer Höhle oder aus deren allernächster Umgebung ${ }^{2}$ ). Der Besitzer bewacht und verteidigt seine neuerworbene Höhle: Sitzt er noch nicht direkt am Eingang, dann rückt er, sobald ein Artgenosse an den Höhleneingang kommt, schnell nach oben und ,schlägt" nach diesem. In ,Wächterstellung “ ist der Körper leicht sichelförmig gebogen (vgl. Abb. 1); beim Schlagen (Abb. 2) wird der Vorderkörper kurz nach hinten oben geworfen. (Hochrücken und Schlagen auslösende Reize sind: leichte Erschütterungen, optische Wahtnehmung von Bewegungen, leichte Berührung an den Tergiten und Fühlern.) Läßt sich die fremde Assel durch vielfaches Schlagen nicht vertreiben, dann verklemmt sich der Wächter (Abb. 3) zwischen den gegenüberliegenden Höhlenwänden. Geschlechtsspezifische Unterschiede im Wächterverhalten konnten nicht festgestellt werden.

\section{Erkennen und Aufsuchen des Geschlechtspartners}

Die in Tab. 1 wiedergegebenen Beobachtungen zeigen, daß sich höhlenlose Asseln intensiv und ausdauernd vorwiegend an solchen Höhlen um Einlaß bemühen, die von einem alleinstehenden, andersgeschlechtlichen Artgenossen besetzt sind.

Erkennen sich die Geschlechtspartner am Verhalten bei ihrer Begegnung am Höhleneingang? Der Eindringling nähert sich dem Wächter langsam und betastet ihn vorsichtig mit den Fühlern ${ }^{3}$ ),

1) Als Aktivitätsphasen werden diejenigen Tageszeiten bezeichnet, während det sich wenigstens $5-10 \%$ der im Beobachtungsgebiet wohnenden Asseln regelmäßig außerhalb ihrer Höhlen aufhalten. Im Frühjahr ist dies meist zwischen 7.30 und 11.00 und von $16.00-18.30 \mathrm{Uhr}$ der Fall.

2) Sie ernähren sich von trockenen Pflanzenteilen, die der Wind an den Eingang geweht hat. Falls die Winddrift nicht für genügend Nahrung sorgt, verlängern sie ihre Höhle und verzehren einen Teil des dabei anfallenden Materials.

3) Hier und im folgenden sind immer die 2. Antennen gemeint. Bei allen Oniscoideen sind die 1. Antennen sehr stark reduziert. 
Tab. 1: Geschlecht der einzelstehenden Höhlenbesitzer bzw. (wenn die Höhle schon von einem Pärchen bewohnt war) der Wächter, bei denen sich Eindringling-ơ oder Eindringling-o zwischen dem 16. - 28. 3. länger als 10 Minuten intensiv um Einlaß bemühten.

Bei Pärchen wird in dieser Tab. der Wächter an erster Stelle geschrieben

\begin{tabular}{|c|c|c|c|c|}
\hline \multirow{2}{*}{$\begin{array}{l}\text { Geschlecht des } \\
\text { Eindringlings }\end{array}$} & \multicolumn{4}{|c|}{ Geschlecht des H.-Besitzers oder Wächters } \\
\cline { 2 - 5 } & $\sigma^{\pi}$ & $q$ & $\sigma^{\pi} q$ & $q \sigma^{\star}$ \\
\hline$\sigma^{\star}$ & 4 & 41 & $2\left(+1^{2}\right)$ & 6 \\
\hline$q$ & 31 & 3 & 12 & 1 \\
\hline
\end{tabular}

a Dieses ô zerrte den Wächter aus der Höhle.

worauf dieser regelmäßig schlägt. Der Eindringling kann schon nach dem ersten Fühlerkontakt mit dem Wächter weggehen und nach einer neuen Höhle suchen. In einem solchen Fall ist der Eindringling fast immer mit dem Wächter gleichgeschlechtlich. Sonst zieht er sich nach dem Schlagen des Wächters nur maximal $2 \mathrm{~cm}$ vom Höhleneingang zurïck. Dort bleibt er einige Sek. oder Min. sitzen (im Extremfall auch Stunden), bevor er den Wächter wieder betastet. Mit zunehmender Zahl der Abweisungen durch den Wächter wird die Wahrscheinlichkeit größer, daß auch ein nicht mit dem Wächter gleichgeschlechtlicher Eindringling abwandert.

Gegenüber einer verschiedengeschlechtlichen Assel, die sich ausdauernd um Einlaß bemüht, ändert der einzelstebende Wächter normalerweise sein Verhalten: Er schlägt mit geringerem Nachdruck, hört schließlich ganz damit auf und klemmt sich nur fest. Der Eindringling beginnt nun - wie beim Graben - mit den vorderen Beinpaaren auf den Peraeontergiten des Wächters zu scharren (Abb. 4). Mit dem Scharren, das in Phasen von wenigen Sek. bis 5 Min. Dauer erfolgt und von sehr verschieden langen Pausen (einige Sek. bis $1 / 2$ Std.) unterbrochen wird, erreicht der Eindringling nach minimal $20 \mathrm{Min}$. bis maximal $14 \mathrm{Std}$. (im Mittel nach $2,1 \pm 1,5$ Std., $n=19$ ), daß ihn der Wächter in die Höhle läßt. Dabei kann dieser entweder aus der Höhle kommen (Abb. 5) oder - bei größerem Innendurchmesser der Höhle - sich flach an die Wand pressen und den Eindringling an sich vorbei in die Höhle lassen. Oder aber er rückt nur etwas tiefer in die Höhle und läßt den Eindringling seine Position und seine Funktion als Wächter übernehmen. Unter natürlichen Bedingungen wird der Eindringling im ersten Fall nie mehr vertrieben. Beim letztgenannten Einlaßmodus dagegen kann er vom alten Höhlenbesitzer wieder verjagt werden (vgl. S. 139).

Das Verhalten von Wächter und Eindringling am Höhleneingang ließ nie regelhafte geschlechtsspezifische Unterschiede erkennen. Auffallend war immer, mit welcher Häufigkeit sich beide gegenseitig mit den Fühlern be-

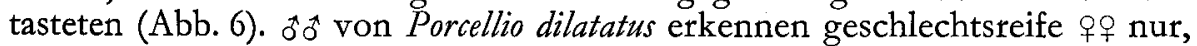
wenn sie sie mit den Fühlerendgliedern berühren (LeGRAND 1958). Bevor die Frage geklärt werden konnte, ob das Fühlerbetasten auch bei $H$. reaumuri etwas mit dem Erkennen des Geschlechtspartners zu tun hat, mußte - als Voraussetzung für die entscheidenden Experimente - zunächst versucht werden, unter experimentellen Bedingungen Paarbildungen zu induzieren:

1. Schüsseln von $30 \times 30 \times 25 \mathrm{~cm}$ wurden $20 \mathrm{~cm}$ hoch mit durchfeuchtetem, festgestampftem Sand gefüllt. In diesen Untergrund bohrten wir 10 leicht schräge, $15 \mathrm{~cm}$ tiefe und $0,9 \mathrm{~cm}$ weite Höhlen. Dann setzten wir in jede Schüssel $15 \delta \sigma^{\star}$ und 15 qo. Die Höhlen wurden notmalerweise von der jeweils ersten Assel, ơ oder o, die auf den Eingang stieß, besetzt und wie eine Höhle im natürlichen Biotop verteidigt. Die Versuche wurden vor Beginn der morgendlichen Aktivitätsphase angesetzt und nach Ende der 2. Aktivitätsphase ausgewertet. In 64 der 74 von 2 Asseln besetzten Höhlen waren je ein $\delta^{*}$ und ein $q$, in 6 waren $2 \delta^{*} \delta^{*}$ und in 42 우. Demnach kommt es auch unter diesen experimentellen Bedingungen zu Paarbildungen. Eine andere Erklärung für die hochsignifikante Bevorzugung eines verschiedengeschlechtlichen Partners erscheint wenig wahrscheinlich.

2. In Gefäßen von $10 \times 10 \times 10 \mathrm{~cm}$ wurden - mit jeweils vier $6 \mathrm{~cm}$ tiefen Höhlen - ähnliche Versuche durchgeführt. Im Gegensatz zu den unter 1. geschilderten Bedingungen setzten wir nun zunächst 2 ơ und 2 웅 in die Höhlen. Nachdem jeder der 4 Höhlenbesitzer 3-6 Std. als Wächter in seiner Höhle verbracht hatte, wurden je Gefäß gleichzeitig 5 ơ $\sigma^{*}$ und 5 qo eingesetzt. In 25 Höhlen mit 2 Insassen, in denen zunächst $\delta^{\dagger} \delta$ Wächter waren, fanden wir $24 \mathrm{mal} \delta, \phi$ und nur einmal $\delta^{\star}, \sigma^{*}$. Fast identisch fiel das Ergebnis in den Versuchen aus, in denen die 우 Wächter waren: $32 \mathrm{mal} \delta$, 우 und $2 \mathrm{mal} \%$, 9 .

Die Tatsache, daß sich beim 1. Versuch mehr gleichgeschlechtliche Paare bildeten, hat aller Wahrscheinlichkeit nach nichts mit der Versuchsmethodik zu tun. Ein Teil dieser Versuche fand bei relativ niedrigen Temperaturen statt $\left(12-15^{\circ} \mathrm{C}\right)$ : Von 27 Paaren waren unter diesen Bedingun- 

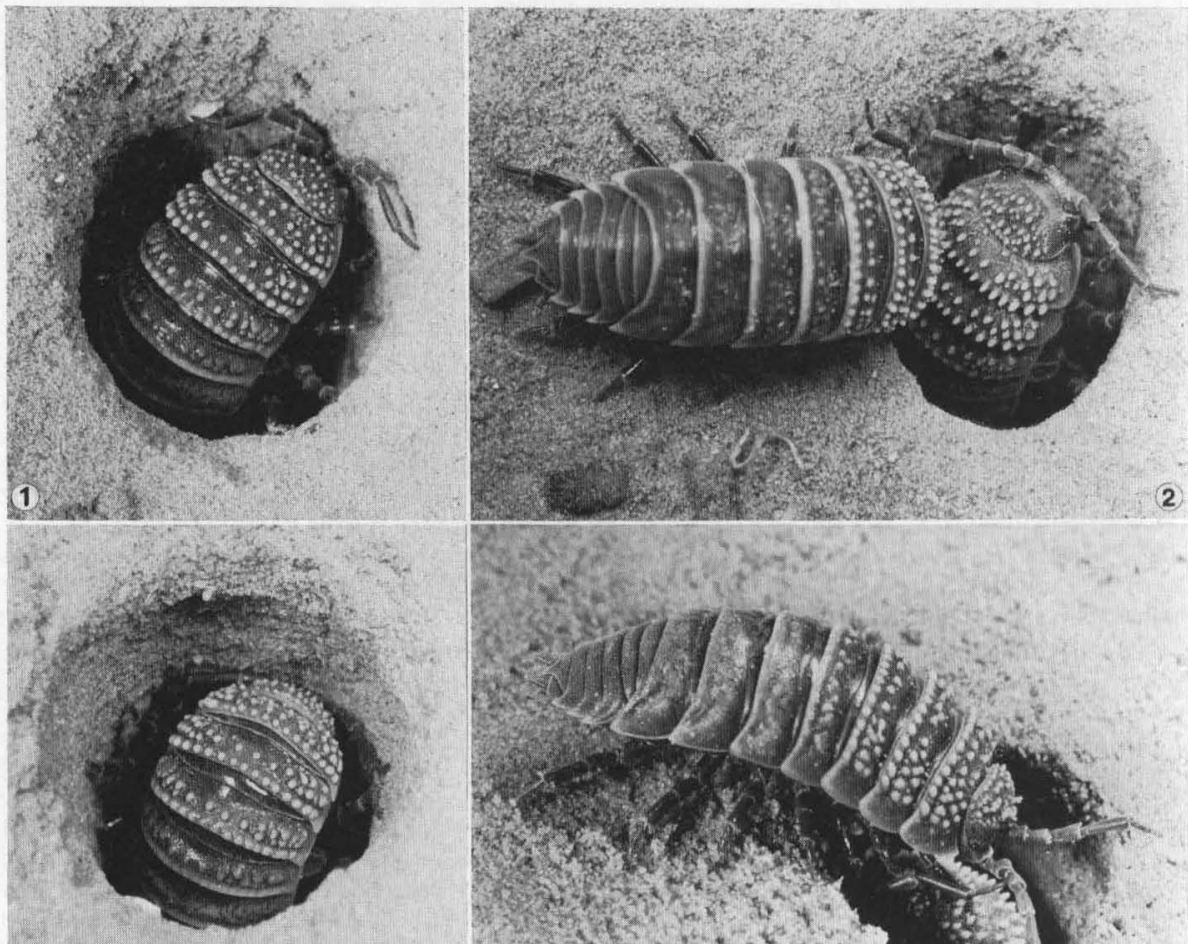

(3)
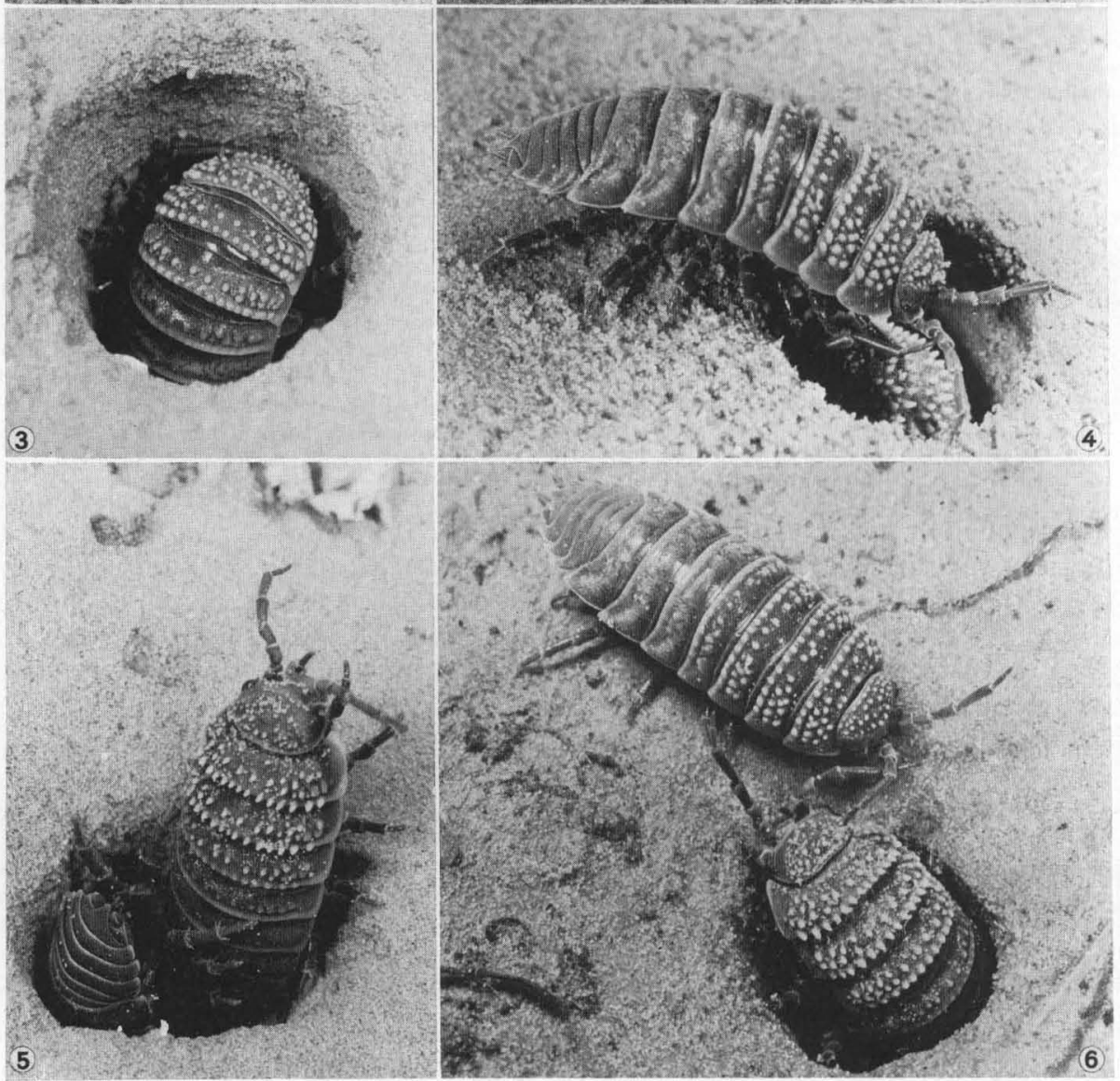

$A b b$ 1: Wüstenassel in Wächterhaltung am Eingang ihrer Höhle

$A b b .2$ : Der Wächter schlägt durch Hochwerfen des Vorderkörpers (vgl. die Ausgangsstellung in $\mathrm{Abb} .1$ ) nach einem fremden Eindringling

$A b b$. 3: Ein Wächter verklemmt sich zwischen den gegenüberliegenden Höhlenwänden. Die kegelförmigen Höcker auf der Kopfoberseite und den vorderen Paraeontergiten dürften sehr wahrscheinlich im Zusammenhang mit der spezifischen Art der Höhlenverteidigung selektioniert worden sein 
gen 7 gleichgeschlechtlich. Gleichartige Versuche bei höheren Temperaturen $\left(20-28^{\circ} \mathrm{C}\right)$ ergaben ein Verhältnis von $44 \hat{\circ}, 9$ zu 3 gleichgeschlechtlichen Paaren. Dies spricht sehr dafür, daß höhlenlose Asseln unter ungünstigen Außenbedingungen in einer Höhle vor allem Unterschlupf und nicht vornehmlich einen Partner suchen. $\mathrm{DaB}$ ein Teil der Wächter ihre Bauten bei ungünstigen Wetterverhältnissen mit geringerer Intensität verteidigt, läßt sich direkt beobachten. Sobald wieder günstigete Witterungsbedingungen herrschen, jagen die Wächter gleichgeschlechtliche Eindringlinge, die sie nie an sich vorbei, sondern nur über sich gelassen haben, immer aus der Höhle.

Diese Versuchsergebnisse erlaubten eine nähere Untersuchung der oben gestellten Frage. Vor allem nach Versuchsmethodik 2 wurden zahlreiche Paarbildungsversuche mit Asseln angesetzt, denen wir die beiden letzten Glieder an jeder Antenne mindestens $24 \mathrm{Std}$. (maximal 6 Tage) vor dem Versuch amputierten: In 122 Fällen fanden wir am Ende des Versuchs ein verschieden-, $118 \mathrm{mal}$ ein gleichgeschlechtliches Paar in einer Höhle. Um nachzuprüfen, ob nicht Verletzungseffekte für dieses Ergebnis ausschlaggebend sind, wurden unter sonst identischen Bedingungen Versuche mit Asseln durchgeführt, deren Fühlerendglieder wir mit farblosem Nagellack oder Emaillelack überzogen: von 49 Paaren waren 22 verschieden- und 27 gleichgeschlechtlich. Auch wenn nur das letzte Fühlerglied (beidseitig) amputiert wurde, konnte die 0-Hypothese: gleiche Wahlhäufigkeit eines gleich- und eines verschiedengeschlechtlichen Partners, nicht verworfen werden (100 Paare, davon 56 verschiedenund 44 gleichgeschlechtlich).

Die Beobachtung, daß es nach Amputation der Fühlerendglieder mit gleicher Wahrscheinlichkeit zur Bildung gleich- und verschiedengeschlechtlicher Paare kommt, spricht für den Wegfall eines gleichgeschlechtlichen Hemmfaktors. Wäre ein - mit Fühlerrezeptoren wahrgenommenes auslösendes Merkmal des andersgeschlechtlichen Artgenossen von entscheidender Wichtigkeit bei der Paarbildung, dann sollte man erwarten, daß die Amputationen Paarbildungen vollständig verhindern.

Amputiert man die Fühlerendglieder nur bei einem Beteiligten, entweder dem Wächter oder dem Eindringling, dann nimmt die Zahl der Fehlpaarungen gegenüber vergleichbaren Versuchen mit intakten Asseln zu: Von 97 Paaren, die am Ende der 2. Aktivitätsperiode nach Versuchsbeginn aus je einer Höhle ausgegraben wurden und bei denen der Wächter amputierte Endglieder hatte, waren 69 verschieden- und 28 gleichgeschlechtlich. Beim umgekehtten Versuch - Wächter mit intakten Fühlern, Eindringlinge mit amputierten Endgliedern - kamen auf 63 verschieden- 15 gleichgeschlechtliche Paare. (Im letztgenannten Fall durfte der amputierte Eindringling nie am Wächter vorbei in die Höhle.)

In beiden Versuchen bildeten sich signifikant mehr verschiedengeschlechtliche Paare. Dies beweist, wie schon auf Grund früherer Beobachtungen (z. B. S. 136) und Versuchsergebnisse zu erwarten war, daß sowohl Wächter als auch Eindringlinge in der Regel Fehlpaarungen vermeiden. (Wie in den vorigen Versuchen (s. S. 137) zeigte sich auch hier wieder, daß sowohl 우우 als auch 숭ㅅ den gleich- vom verschiedengeschlechtlichen Artgenossen unterscheiden.)

Die Ursachen der erhöhten Zahl von Fehlpaarungen liegen auf der Hand: Der amputierte Wächter erkennt das Geschlecht des Eindringlings nicht mehr. Jeder, der sich intensiv und lange genug um Einlaß bemüht (wenn er z. B. wegen ungünstiger Außenbedingungen Unterschlupf sucht),

\section{Fortsetzung der Bildunterschrift von Seite 138}

Abb. 4: Sobald der Wächter nicht mehr schlägt, scharrt der Eindringling mit den 4 vorderen Beinpaaren auf Kopf und Peraeontergiten des sich verklemmenden Wächters

Abb. 5: Der Wächter läßt seinen Partner an sich vorbei, indem er ein Stück weit aus der Höhle kommt und sich außerdem "flach macht"

Abb.6: Bei jeder Begegnung am Höhleneingang betasten sich die ankommende und die wachehaltende Assel 
wird wie ein andersgeschlechtlicher Partner behandelt und in die Höhle eingelassen. Umgekehrt erkennt der amputierte Eindringling nicht mehr das Geschlecht des Wächters: Einzelne amputierte lassen sich deshalb viel weniger leicht von einem gleichgeschlechtlichen Wächter vertreiben ${ }^{4}$ ) als intakte Eindringlinge (vgl. aber S. 145).

Es handelt sich immer nur um vorübergehende Fehlpaarungen: Je nachdem, ob der intakte Partner zu Beginn der nächsten Aktivitätsphase selbst Wächter ist oder unter dem Wächter im Höhleninneren sitzt, verläßt er die Höhle freiwillig oder vertreibt den gleichgeschlechtlichen Artgenossen aus der Höhle.

An den Körperstellen, die Wächter und Eindringling bei ihrer Begegnung am Höhleneingang betasten, konnten keine geschlechtsspezifischen morphologischen Unterschiede entdeckt werden. Es scheint deshalb gerechtfertigt, anzunehmen, daß es chemische Eigenschaften (= Pheromone) sind, an denen sich die Geschlechtspartner erkennen. Daß sich auf den Fühlerendgliedern der Oniscoidea Chemorezeptoren befinden, darauf weisen u. a. Verhaltensuntersuchungen von FischBaCH (1954) und Cloudsley-Thompson (1956b) an Oniscus asellus und Porcellio scaber hin.

Die Unterscheidung des gleich- vom verschiedengeschlechtlichen Artgenossen ist nicht auf die Situation der Begegnung von Eindringling und Wächter am Höhleneingang beschränkt. Setzt man während der Paarbildungszeit 15-30 Asseln in ein enges Gefäß (in den Versuchen $10 \times 10 \mathrm{~cm}$ Grundfläche), dann greifen regelmäßig einzelne Asseln Artgenossen an. Sie versuchen, die angegriffene Assel von oben mit den Peraeopoden zu packen, drehen sich dann im Erfolgsfall so, daß ihre Körperlängsachse quer zu der der ergriffenen steht, wobei sie sich normalerweise auf den Rücken fallen lassen. Falls sich die Angegriffene nicht befreien kann, werden ihr Beine abgebissen, manchmal auch Löcher in den Körper genagt. Aggressive Asseln betasten in der Versuchssituation zwar jeden Artgenossen, dem sie begegnen, an der Körperstelle, die zufällig im Bereich ihrer Fühlerspitzen liegt, wie Tab. 2 zeigt, greifen sie aber signifikant bevorzugt gleichgeschlechtliche Artgenossen $\mathrm{an}^{5}$ ). Bei 35 registrierten Überfällen Amputierter war $16 \mathrm{mal}$ eine Assel gleichen Geschlechts und $19 \mathrm{mal}$ eine verschiedengeschlechtliche die Angegriffene. Eine Unterscheidung der Geschlechter ist also auch unter diesen Bedingungen nach einer Amputation der Fühletendglieder nicht mehr möglich.

Man findet immer wieder Eindringlinge, die mit einigem Zeitaufwand (10-15 Min.) versuchen, in die Höhlen gleichgeschlechtlicher Artgenossen zu gelangen. Sie bemühen sich, entweder vorübergehenden Unterschlupf zu finden (s. S. 137) oder den Wächter zu vertreiben. Zu letzterem veranlassen sie vor allem Wächter, die weder heftig schlagen, noch sich richtig festklemmen; es sind also entweder sehr kleine oder geschwächte Exemplare.

Die Assel, die sich eine Höhle gegraben oder eine leere angeeignet hat, sitzt als Wächter in ihrem Bau und ,wartet", bis sich ein Geschlechtspartner um Einlaß bemüht. Was geschieht, wenn während einer längeren Zeitspanne kein Artgenosse an die Höhle kommt? In einem etwa $25 \mathrm{qm}$ umfassenden Gebiet wurden aus 20 Höhlen die Insassen entfernt. In diese Höhlen setzten wir markierte Asseln (11 우우 und $9 \overrightarrow{0} \widehat{0})$, die zuvor mindestens $72 \mathrm{Std}$. isoliert in kleinen Gefäßen oder künstlichen Höhlen gehalten worden waren. Während der Aktivitätszeiten in den folgenden 4 Tagen wurde das Gebiet kontinuierlich überwacht, jede fremde, höhlenlose Assel abgefangen und jede Außenaktivität der markierten Asseln beobachtet. Die wichtigsten Ergebnisse sind:

4) Vertreibung heißt: 1 . Der Eindringling geht infolge des heftigen Schlagens des Wächters weg und sucht eine neue Höhle. 2. Der Eindringling wird durch den Wächter, der aus der Höhle gekommen ist, direkt aus der Höhlenumgebung verjagt.

5) Unter natürlichen Bedingungen greifen sich gleichgeschlechtliche Asseln dann an, wenn 2 gleichzeitig versuchen, in dieselbe Höhle einzudringen. 
Tab. 2: Geschlechtsverhältnis von kämpfenden Asseln (s. Text)

\begin{tabular}{|c|c|c|}
\hline \multirow{2}{*}{ Angreifer } & \multicolumn{2}{|c|}{ angegriffen } \\
& $\wp$ & $0^{\pi}$ \\
\hline$q$ & 66 & 5 \\
\hline 8 & 8 & 38 \\
\hline
\end{tabular}

1. Innerhalb der ersten $36 \mathrm{Std}$. (=4 Aktivitätsphasen) verließen nur 2 ofo und $2{ }^{*}{ }^{*}$ für einige Min. ihre Höhlen und beschafften sich in 20-60 cm Entfernung von den Eingängen Nahrung.

2. In den folgenden $48 \mathrm{Std}$. beobachteten wir 3 o $^{\star}{ }^{*}$ und 5 우, die die nähere Umgebung ihrer Höhlen systematisch ab-,,suchten“. Nahrung ließen sie dabei unbeachtet. Dagegen wurde jede fremde Höhle, auf die sie stießen, inspiziert.

3. Die ersten 2-4 Ausflüge dieser Asseln führten nur 10-30 cm vom Höhleneingang weg und endeten nach 3-12 Min. wieder an der eigenen Höhle. Bei den folgenden Ausflügen entfernten sie sich bis zu $110 \mathrm{~cm}$ und bis zu 40 Min. von ihrer Höhle.

4. Asseln, die bei den Ausflügen auf leere Höhlen stießen, verließen sie nach einer höchstens wenige Minuten dauernden Inspektion immer, kehrten wieder zur eigenen Höhle zurück oder setzten den Ausflug fort.

5. Sobald die Asseln bei ihrem Umherwandern eine Höhle mit einem alleinstehenden andersgeschlechtlichen Artgenossen fanden, bemühten sie sich - wie ein höhlenloser Eindringling - um Einlaß. Sobald eine Assel bei einem Partner eingelassen wurde, kehrte sie niemals mehr zu ihrer alten Höhle zurück.

6. Asseln, die bei Ausflügen in der näheren Umgebung keine Höhle mit Partner fanden, entfernten sich endgültig von ihrer Höhle und wurden in ihrem Verhalten wieder zu höhlenlosen Eindringlingen.

7. 84 Std. nach dem Versuchsbeginn hatten bereits 2 హิ und 3 우 in benachbarten Höhlen einen Partner gefunden. Sechs Tage nach Versuchsbeginn befanden sich von 11 ofo nur noch 2 und von den $90^{\star} 0^{\star}$ ebenfalls nur noch 2 allein in ihren Höhlen. Alle übrigen waren abgewandert.

Diese Beobachtungen lassen keine Zweifel daran, daß bei den Ausflügen ein Geschlechtspartner "gesucht" wird. Um die Suchgänge auszulösen, bedarf es nach den Ergebnissen dieser Experimente Ende März einer sich über wenigstens 4 Aktivitätsphasen erstreckenden Zeitspanne der vollständigen Isolation von Artgenossen.

Es muß aber unbedingt beachtet werden, daß die Asseln schon vor Versuchsbeginn mindestens 3 Tage isoliert gehalten worden waren und ihre Höhle dann ohne jedes Suchen fanden. Bei einer Assel, die eine leere Höhle gefunden hat, nachdem sie längere Zeit höhlenloser Eindringling war, bedarf es sehr wahrscheinlich einer längeren Isolationszeit; dasselbe dürfte während dieser Jahreszeit für Asseln gelten, die sich selbst eine Höhle gebaut haben.

\section{Paarzusammenhalt}

Sofort nachdem der Wächter einen verschiedengeschlechtlichen Artgenossen in die Höhle eingelassen hat, wehrt er jeden neuen Eindringling - unabhängig von dessen Geschlecht und der Dauer und Intensität seiner Bemühungen - $a b$, auch dann, wenn sich sein Partner zur Nahrungssuche außerhalb der Höhle befindet. Seinen Partner dagegen läßt er sofort in die Höhle, wenn die beiden schon mehrere Std. bis einen Tag gemeinsam in 
der Höhle verbracht haben (s. S. $146 \mathrm{ff}$.), und wenn er ihn mit den Fühlern berührt hat. In vielfach wiederholten Versuchen zeigte sich immer wieder, daß von bis zu 100 fremden, an den Höhleneingang gesetzten Asseln nie eine mit dem Partner verwechselt und wie er in die Höhle eingelassen wurde. Diese Beobachtungen legen die Annahme nahe, daß der Wächter seinen Partner individuell kennt.

\section{Das individuelle Erkennen des Partners}

Ein Eindringling nähert sich der Höhle vorsichtig und betastet den Wächter zögernd mit den Fühlern. Eine verpaarte Assel dagegen, die nach einem Ausflug in ihre Höhle zurückkehrt, rennt ohne Zögern gegen den Wächter an, falls dieser ihr nicht Platz macht. Danach könnte man vermuten, daß der Partner in Wirklichkeit doch nicht individuell erkannt wird, sondern nur am „sicheren Auftreten“, das er an seiner eigenen Höhle zeigt. Folgende 3 Experimente (jeweils $n=20$ Einzelversuche) sollten diese Frage entscheiden:

1. Ein Pärchen wird aus seiner Höhle ausgegraben. Frühestens $2 \mathrm{Std}$. danach wird ein Partner an eine künstliche Höhle gebracht, die er in der Regel sofort besetzt. Einige Minuten nachdem er sich umgedreht hat und mit dem Kopf zum Eingang sitzt (= die Wächterposition eingenommen hat), witd sein Partner dazugesetzt. Dieser nähert sich der ganz offensichtlich als fremd erkannten Höhle sehr vorsichtig. Sitzt der Wächter einige $\mathrm{cm}$ unterhalb des Eingangs, kann es vorkommen, daß sich sein Partner nur ganz kurz mit vibrierenden und kreisenden Bewegungen der Fühler in die Höhle beugt und dann weggeht. Berührt er den Wächter aber beim vorsichtigen Tasten mit den Fühlerspitzen, dann kriecht er immer in die Höhle. Er wird vom Wächter, sobald dieser ihn seinerseits betastet hat, ausnahmslos und ohne Abwehr eingelassen. Fremde Asseln, die sich an der Versuchshöhle nicht unsicherer benehmen als der Paarpartner, werden innerhalb der ersten Stunden immer nachdrücklich und erfolgreich abgewiesen.

2. Setzt man einen Partner eines ausgegrabenen Pärchens an eine fremde Höhle, deren Insassen man unmittelbar vor dem Versuch entfernt hat, dann wird diese Höhle - immer deutlich zögernd - von der Mehrzahl der Versuchstiere besetzt. (Voraussetzung ist allerdings, daß man die Pärchen, mit denen die Versuche angestellt werden, schon wenigstens $1 / 2$ Tag vorher aus ihrer Höhle entfernt hat. Unmittelbar nach dem Herausfangen aus der Höhle sind mehr als die Hälfte der Versuchstiere nicht zu bewegen, freiwillig in eine zuvor von fremden Asseln bewohnte Höhle zu kriechen.) Bringt man nun die zweite Assel an den Eingang der fremden Höhle, in der ihr Partner als Wächter sitzt, dann beobachtet man eine oft noch sehr viel unsicherere Annäherung als im ersten Versuch. Sobald aber die Assel am Höhleneingang ihren Partner mit den vorsichtig tastenden Fühlerspitzen berührt, kriecht sie in die Höhle, was ausnahmslos vom Wächter geduldet wird.

3. Hier wird Versuch 2 wiederholt. An Stelle des eigenen Partners wird aber im kritischen Experiment die im Geschlecht entsprechende Assel aus der Versuchshöhle dazugesetzt ${ }^{6}$ ). Im Gegensatz zum Partner des Wächters läuft diese Assel schnell, ohne jede Unsicherheit und ohne zögerndes Fühlertasten in die Höhle. Trotzdem wird sie immer sehr energisch abgewehrt.

6) Es wurden nur solche Asseln verwendet, die bei einem Ausflug gefangen wurden. Zieht man die Assel z. B. mit einer Pinzette aus ihrer Höhle, dann kann sie häufig nicht mehr dazu bewegt werden, ihre eigene Höhle nochmals aufzusuchen. Sie reagiert so, als ob sie ein Artgenosse aus der Höhle gezerrt hätte: Auch dann verläßt sie meist fluchtartig die Höhlenumgebung und bemüht sich nach unseren Beobachtungen nie mehr um eine Wiedereroberung. 
Diese Versuchsergebnisse lassen zwei wichtige Schlußfolgerungen zu:

1. Es ist nicht das ,sichere Auftreten“ der heimkommenden Assel, das den Wächter veranlaßt, sie passieren zu lassen. Er müßte sonst in Versuch 3 die fremde Assel in die Höhle lassen und im Versuch 2 den eigenen Partner abweisen. Im 1. Versuch dürfte er weder den eigenen Partner noch eine sich nicht weniger unsicher benehmende fremde Assel einlassen oder aber beide mit der gleichen Wahrscheinlichkeit. Es muß sich vielmehr um eine vom Verhalten des Partners unabhängige Eigenschaft handeln.

2. Das Erkennen des Partners ist weder beim Wächter noch beim von außen kommenden Partner an die eigene Höhle gebunden: Jeder erkennt seinen Paarpartner auch in einer in unbekannter Umgebung liegenden künstlichen oder natürlichen, aber fremden Höhle.

Reaktionen, die auf ein Erkennen des Partners schließen lassen, treten immer erst nach dem Betasten mit den Fühlern auf. Dies spricht dafür, daß Antennenrezeptoren das individuelle Erkennen des Partners ermöglichen. Amputationsversuche sollten diese Vermutung unterstützen oder widerlegen.

Vorversuch: 11 Pärchen wurden aus ihren Höhlen ausgegraben und je 1 Partner an eine künstliche Höhle gesetzt, die er immer bezog. Nach 40 bis 44 Std. wurde geprüft, wie die Höhlenbesitzer auf ihre eigenen Partner und fremde, mit dem Partner gleichgeschlechtliche Asseln reagierten. Die Ergebnisse sind in der Tab. 3 zusammengefaßt. Der Vorversuch zeigt, daß auch nach 40-44 Std. der eigene Partner noch eindeutig von fremden Asseln unterschieden wird.

Tab. 3: Reaktionen von 11 intakten Wächtern (die 40-44 Stunden allein in einer Höhle saßen) auf ihren Partner und auf fremde Asseln, die in die Höhle einzudringen versuchten. An 4 Höhlen wurden nacheinander 2 Eindringlinge gesetzt

\begin{tabular}{|c|c|c|c|c|}
\hline Eindringling & $n$ & $\begin{array}{l}\text { wieviele } \\
\text { eingelassen a }\end{array}$ & $\begin{array}{l}\text { Dauer der Bemühungen vom } \\
\text { ersten Kontakt bis zum } \\
\text { Einlaß }\end{array}$ & $\begin{array}{l}\text { bei wievielen Eindringlingen } \\
\text { schlägt der Wächter trotz } \\
\text { vorausgehendem Fühler - } \\
\text { betasten }\end{array}$ \\
\hline $\begin{array}{l}\text { eigener } \\
\text { Partner }\end{array}$ & 11 & 11 & 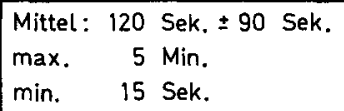 & $0^{b}$ \\
\hline $\begin{array}{l}\text { fremder } \\
\text { Eindr ingling }\end{array}$ & 15 & 2 & $\begin{array}{l}25 \text { Min. } \\
32 \text { Min. }\end{array}$ & bei allen 15 \\
\hline
\end{tabular}

a Eine Assel galt in diesem Experiment als nicht eingelassen, und damit als fremd erkannt, wenn sie sich länger als $35 \mathrm{Min}$. erfolglos um Einlaß bemühte oder wenn sie 3 mal von der Höhle vertrieben wurde (vgl. Fußnote 4).

b Die Wächter schlagen zwar nicht, aber einige verklemmen sich trotz vorausgehendem Betasten und machen ihrem Partner erst auf dessen Scharren hin (vgl. S. 137) nach maximal 5 Min. Platz.

1. Hauptversuch (Tab. 4a): Allen beteiligten Asseln wurden die beiden Endglieder an jedem Fühler entfernt. Zwischen Amputation und dem erneuten Zusammensetzen der Partner ließen wir 5-30 Std. verstreichen. Wir fanden keine Anhaltspunkte dafür, daß das Verhalten noch 5 Std. nach dem Eingriff durch unspezifische, operationsbedingte Verletzungseffekte beeinflußt wird. Stichproben, bei denen die Fühlerspitzen nicht amputiert, sondern lackiert worden waren, erbrachten nie differierende Ergebnisse. Die längste. Trennungszeit von 30 Std. führt nach den Ergebnissen des Vorversuchs zu keinen erheblichen, zeitlich bedingten Störungen des Wiedererkennens. 
Tab. 4: Reaktionen von amputierten Wächtern (a, b, d) auf ihre ebenfalls amputierten Partner und fremde Eindringlinge $(\mathrm{a}, \mathrm{b}) \mathrm{bzw}$. auf intakte Partner und intakte fremde Eindringlinge. In Versuch $\mathrm{c}$ hatten die Wächter intakte Antennen, ihre Partner und fremde Eindringlinge amputierte (s. Text, vgl. auch Tab. 3). Eine vertriebene Assel wird noch weitere $4 \mathrm{mal}$ an die Höhle gesetzt

\begin{tabular}{|c|c|c|c|c|c|c|}
\hline & $\begin{array}{l}\text { Eindring- } \\
\text { ling }\end{array}$ & $n$ & $\begin{array}{l}\text { wieviele } \\
\text { eingelassen }\end{array}$ & $\begin{array}{l}\text { Dauer der } \\
\text { Bemühungen }\end{array}$ & $\begin{array}{l}\text { Schlagen } \\
\text { trotz } \\
\text { Fühlerbetasten }\end{array}$ & $\begin{array}{l}\text { Eindringling "riecht" } \\
\text { nur kurz in Höhle u. } \\
\text { geht ohne Eindring- } \\
\text { versuch weg }\end{array}$ \\
\hline \multirow{2}{*}{$\mathbf{a}$} & $\begin{array}{l}\text { eigener } \\
\text { Partner }\end{array}$ & 18 & $12 \quad(11)^{a}$ & $\begin{array}{ll}\text { Mittel: } 5 \pm 4 \text { Min. } \\
\text { max.: } 11 \text { Min. } \\
\text { min.: } 25 \text { Sek. }\end{array}$ & 8 & 3 \\
\hline & $\begin{array}{l}\text { fremde } \\
\text { Assel }\end{array}$ & 24 & $19 \quad\{18\}$ & $\begin{array}{ll}\text { Mittel: } & 4 \pm 4 \text { Min. } \\
\text { max.: } & 18 \text { Min. } \\
\text { min.: } & 10 \text { Sek. }\end{array}$ & 13 & 2 \\
\hline \multirow{2}{*}{ b } & $\begin{array}{l}\text { eigener } \\
\text { Partner }\end{array}$ & 9 & $7 \quad(7)$ & $\begin{array}{ll}\text { Mittel: } & 4,5 \pm 2 \text { Min. } \\
\text { max.: } & 7 \text { Min. } \\
\text { min.: } & 15 \text { Sek. }\end{array}$ & 3 & 1 \\
\hline & $\begin{array}{l}\text { fremde } \\
\text { Assel }\end{array}$ & 7 & $5 \quad(5)$ & $\begin{array}{ll}\text { Mittel: } & 5,5 \pm 5 \text { Min. } \\
\text { max.: } & 12 \text { Min. } \\
\text { min.: } & 75 \text { Sek. }\end{array}$ & 4 & 1 \\
\hline \multirow{2}{*}{ c } & $\begin{array}{l}\text { eigener } \\
\text { Partner }\end{array}$ & 16 & $14 \quad(6)$ & $\begin{array}{ll}\text { Mittel: } & 15 \pm 12 \text { Min. } \\
\text { max.: } & 35 \text { Min. } \\
\text { min.: } & 75 \text { Sek. }\end{array}$ & 0 & 2 \\
\hline & $\begin{array}{l}\text { fremde } \\
\text { Assel }\end{array}$ & 31 & 0 & - & 31 & 0 \\
\hline \multirow{2}{*}{ d } & $\begin{array}{l}\text { eigener } \\
\text { Partner }\end{array}$ & 9 & $7^{b} \quad(7)$ & $\begin{array}{l}\text { Mittel: } 1,5 \pm 5 \text { Min. } \\
\text { max.: } 7,5 \text { Min. } \\
\text { min.: } 7 \text { Sek. }\end{array}$ & 5 & 0 \\
\hline & $\begin{array}{l}\text { fremde } \\
\text { Assel }\end{array}$ & 15 & $6 \quad(6)$ & $\begin{array}{ll}\text { Mittel: } & 1 \pm 1 \text { Min. } \\
\text { max.: } & 2,5 \text { Min. } \\
\text { min.: } & 20 \text { Sek. }\end{array}$ & 8 & 6 \\
\hline
\end{tabular}

a In Klammer angegeben: Zahl der Asseln, die beim ersten Versuch eingelassen werden.

b Die beiden restlichen Asseln ließen sich nicht von ihrem Partner vertreiben, es dauerte aber 1 bzw. $21 / 2$ Stunden, bis sie eingelassen wurden.

2. Hauptversuch (Tab. 4b): Den beteiligten Asseln amputierten wir an jedem Fühler nur jeweils das letzte Fühlerglied.

3. Hauptversuch (Tab. 4c): In diesem Experiment besaßen die Wächter intakte Fühler; bei ihren Partnern und fremden Eindringlingen wurden die beiden Endglieder an jeder Antenne amputiert.

4. Hauptversuch (Tab. 4d): Im Gegensatz zum vorigen Versuch entfernten wir beim Wächter die beiden Endglieder der Antennen, sein Partner und fremde Eindringlinge hatten intakte Fühler.

Nach der Amputation der Fühlerendglieder (Tab. 4a u. b) erkennen sich die Partner nicht mehr: Der einzelstehende Wächter läßt eine fremde Assel mit der gleichen Bereitschaft und Wahrscheinlichkeit in die Höhle, wie seinen Partner. Ein Wächter, der nach einer fremden Assel schlägt, schlägt auch nach seinem Partner; schlägt er nicht nach seinem Partner, dann unterläßt er diese Abwehr auch gegenüber fremden Artgenossen. Im 3. Hauptversuch zeigt sich, daß ein Wächter mit intakten Fühlern erwartungsgemäß fremde Asseln, die ausnahmslos innerhalb von 35 Min. nicht in die Höhle durften, vom eigenen 
Partner unterscheidet. Es tritt aber auch bei Direktbeobachtungen mit aller Deutlichkeit zutage, wie wichtig es ist, daß auch der Partner außerhalb der Höhle den Wächter erkennt: Schlägt letzterer nach dem amputierten Partner (Ursache: er hat ihn noch nicht betastet), dann läßt dieser sich nicht selten vertreiben (vgl. Tab. 4c: nur 6 von 14 werden beim ersten Eindringversuch gleich betastet und dann ohne Abwehr eingelassen; die Amputation macht allerdings nicht jede Assel bei ihren Eindringversuchen ,zaghafter", vgl. S. 140). Ein intakter Partner würde sich in der gleichen Situation nicht abweisen lassen.

Ein Wächter, der seinen amputierten Partner betastet, schlägt - auch wenn dieser sich noch so unsicher benimmt, vgl. S. $142 \mathrm{ff}$. - nie nach ihm. Vielmehr macht er ihm meist durch nachunten-Rücken Platz. Kommt der Partner nun nicht in die Höhle nach, dann beobachtet man häufig folgende Verhaltensweisen des Wächters: Er kommt aus seiner Höhle, stößt den Partner mit den Fühlern an, geht dann tief in die Höhle und bleibt einige Zeit dort unten. Kommt der Partner immer noch nicht nach, geht er wieder aus der Höhle, das Anstoßen wiederholt sich und manchmal benagt er mit den Mandibeln leicht die Fühler seines Partners. Dann bleibt er neben dem Eingang stehen oder geht wieder tief in die Höhle. Dies kann sich mehrfach wiederholen, bis der Partner diesen "Aufforderungen" Folge leistet.

Auch der letzte Hauptversuch beweist, wie wichtig es für den Paarzusammenhalt ist, daß beide Partner sich gegenseitig erkennen: Von den 15 fremden intakten Asseln, die an Höhlen Amputierter gesetzt wurden, versuchten 9 in die Höhle zu kommen, 6 gelang es nach 20 Sek. bis nur maximal $21 / 2$ Min. Dauer; sie wurden ganz offensichtlich mit dem eigenen Partner verwechselt. Vier erfolgreiche fremde Eindringlinge warfen daraufhin den Wächter sofort und endgültig aus seiner Höhle.

Es bleibt noch zu erwähnen, daß eine intakte Antenne zum einwandfreien Erkennen des Partners ausreicht.

Zusammenfassend ergeben die Amputationsversuche folgende Resultate:

1. Das zweifelsfrei vorhandene individuelle Erkennen des Paarpartners geht mit der Amputation der Endglieder an beiden Antennen verloren.

2. Ein Paarzusammenhalt über längere Zeit ist nur möglich, wenn beide ihren Partner von fremden Asseln unterscheiden können. Irrt sich der Wächter und läßt eine fremde Assel ohne langdauernde Einlaßprozedur in die Höhle, dann läuft er Gefahr, aus seiner eigenen Höhle geworfen zu werden. Erkennt der von außen kommende Partner im Wächter seinen Partner nicht, kann es vorkommen, daß er überhaupt nicht versucht, in die Höhle zu gelangen oder aber er läßt sich beim ersten Versuch durch ein Schlagen des Wächters, der den Ankömmling durch Betasten noch nicht als seinen Partner identifiziert hat, vertreiben.

Wie beim Erkennen des Geschlechtspartners könnten beim individuellen Erkennen des Paarpartners taktile oder chemische Reize als entscheidende Information von den Fühlerrezeptoren wahrgenommen werden. Das individuelle Erkennen setzt eine sehr hohe Variabilität der relevanten Merkmale voraus. Da zudem - wie beim Erkennen des Geschlechtspartners - die Berührung an einer beliebigen Körperstelle genügt, scheint es sehr wahrscheinlich, daß es sich um chemische Eigenschaften handelt.

Ein direkter Beweis - Entfremden durch Beduften oder teilweises Lackieren eines Partners - ist bislang nicht geglückt. Asseln, die z. B. in kleinen geschlossenen Gefäßen 12-36 Std. sehr starken Duftkonzentrationen von Geraniol, Phenylaethylalkohol, Methylheptenon, Citronellol, Eugenol ausgesetzt waren, wurden von ihrem Partner ausnahmslos erkannt 7 ). Ebenso

7) $\mathrm{DaB}$ die verwendeten Düfte wahrgenommen werden, zeigten Versuche im Zusammenhang mit Fragen der Nahorientierung zum Höhleneingang. 
verliefen Versuche, in denen auf die vorderen Peraeontergite Emaille- oder Nagellack aufgetragen wurde; und ebenso solche, in denen ein Partner 2 bis 3 Tage lang in eine, bis zum Versuchsbeginn von anderen Asseln bewohnte Höhle gesetzt wurde oder in einem sehr engen Gefäß mit einer oder mehreren fremden Asseln zusammenlebte.

Über die Natur der vermuteten individuellen chemischen Merkmale läßt sich damit nicht meht sagen, als daß sie gegenüber Umwelteinflüssen relativ stabil sein müssen. Wie die obigen Versuche (s. S. 143 ergaben, erkennen verpaarte Asseln nach einer Trennungszeit von 40-44 Std. ihren Partner ausnahmslos. In weiteren Versuchen, in denen die Trennungszeit stufenweise verlängert wurde, zeigte sich, daß noch erheblich längere Trennungen zu keinen wesentlichen Störungen beim Wiedererkennen führen ${ }^{8}$ ):

Nach einer Trennungszeit von z. B. 96 Std. erkannten sich die Partner sämtlicher 12 getesteter Paare noch.

Um zu gewährleisten, daß es nur die individuellen Eigenschaften des Partners sind, die ein Wiedererkennen ermöglichen, wurden die Versuche nach der folgenden Methode durchgeführt: Eine Assel wurde allein in ein Gefäß mit künstlicher Höhle gesetzt, die andere während der Trennung in ein kleines Plastikgefäß. Bei jeder höhlenbesitzenden Assel wurde dann, im zuletzt angeführten Fall 96-98 Std, nach der Trennung, geprüft, wie sie auf fremde - selbstverständlich mit dem Partner gleichgeschlechtliche - Asseln reagierte. Eine Assel galt in diesen Versuchen als fremd erkannt, wenn sie der Wächter so oft oder so energisch vertrieb, daß sie nicht mehr dazu zu bewegen war, einen nochmaligen Eindringversuch zu unternehmen oder wenn sie - was die Regel war (vgl. hierzu S. $148 \mathrm{ff}$.) - zwar nicht vertrieben wurde, sich aber ohne Erfolg bereits 20 Min. um Einlaß bemüht hatte. Von den zwölf 96-98 Std. getrennten Paaren ließ der jeweilige Wächter $(6$ of und 6 우) keine der beiden an jede Höhle gesetzten fremden Asseln innerhalb von 20 Min. in die Höhle. Der eigene Partner dagegen durfte immer, sobald er betastet war, sofort oder mit maximal 8 Min. dauernder Verzögerung in die Höhle.

Selbst nach einer Trennungszeit von 29-30 Tagen kannten sich offensichtlich bei 3 von 5 Paaren die Partner noch.

Beide waren während der Trennungszeit in kleinen Plastikgefäßen gehalten worden. 24 bis $48 \mathrm{Std}$. vor dem Zusammensetzen wurde einer Assel eine künstliche Höhle geboten, die sie immer bezog. Von drei Wächtern wurde der Partner nach 45,59 und 90 Sek. in die Höhle eingelassen. Beim vierten Paar stürzte sich das $\delta$, sobald es mit seinen Fühlern das 9 berührt hatte, in die Höhle. Es nahm sofort Wächterposition ein. Das $q$ saß etwas tiefer in der Höhle und rückte beim Erscheinen des ${ }^{\star}$ nicht gleich nach oben. In den nächsten Minuten schob ihn das $q$ aus der Höhle. Nach vierstündigen hartnäckigen Bemühungen durfte das ơ schließlich endgültig in die Höhle. Hicr schien das $\delta$ sein ㅇ noch zu erkennen, dagegen nicht das ? sein $\delta$. Das Umgekehrte beobachteten wir beim fünften Paar. Hier ließ das wachehaltende $q$ sein $\delta$ nach einigen Minuten in die Höhle, worauf dieses sein 9 hinauswarf und innerhalb der nächsten Stunden nicht mehr in die Höhle ließ. Kontrolltiere, die ebensolange von Artgenossen isoliert und dann in eine künstliche Höhle gesetzt worden waten, ließen nie einen fremden Artgenossen innerhalb der ersten zwei Stunden in ihre Höhle.

Die Frage, ob sich in den Fällen, in denen ein Partner den anderen nicht mehr erkennt, die entscheidenden Merkmale geändert haben, oder ob Vergessensprozesse die Ursache sind, läßt sich derzeit nicht beantworten.

\section{Das Sich-Kennenlernen der Partner}

Wann lernen sich die Partner kennen? Wie lange dauert es, bis sie sich kennen und als Folge davon aggressive Handlungen unterdrücken? Etwa $50 \%$ der neu in eine Höhle eingelassenen Asseln beginnen noch während derselben Aktivitätsphase an der Höhle zu graben. Wenn eine dieser Asseln mit dem losgegrabenen Material beim Rückwärtslaufen an das Hinterende des Wächters stößt, macht ihr dieser immer Platz, indem er gerade soweit aus der Höhle kriecht, daß sie an ihm vorbeilaufen kann. Der Wächter geht sofort zurück und nimmt seinen alten Platz wieder ein. Kommt nun sein Partner,

8) Die of 0 der in diesen Experimenten untersuchte Paare waren zum Zeitpunkt der Trennung immer bereits trächtig. 
der das Baumaterial in einer Entfernung von 2-10 cm vom Eingang wegwarf, wieder an den Eingang, dann darf er praktisch nie sofort in die Höhle. Nicht selten dauert es länger als 1 Std., bis der Wächter wieder aus der Höhle kommt oder so weit nach unten rückt, daß sich sein Partner als Wächter über ihn setzen kann. Auch bei den folgenden Begegnungen am Höhleneingang, macht der Wächter erst nach längerem Scharren (s. S. 137) Platz. Nach 3- bis 7maliger Wiederholung der Einlaßprozedur kennt der Wächter seinen Partner offensichtlich; er läßt ihn dann ohne Abwehr in die Höhle.

Der Eindringling lernt den Wächter im Laufe der ersten Einlaßprozesse besser kennen als der Wächter ihn: Man kann den Wächter, gleich nachdem er den Eindringling erstmalig in die Höhle eingelassen hat, durch Schieben von unten (mit einer gebogenen Nadel, die das Anstoßen durch den Partner im Höhleninneren vortäuscht) aus seinem Bau holen, ohne seine Bindung an die Höhle negativ zu beeinflussen (s. Fußnote 6). Hält man ihn außerhalb der Höhle kurzzeitig fest, dann rückt inzwischen der neu eingelassene Eindringling nach oben und nimmt die Wächterposition ein. Läßt man nun den alten Wächter los, dann macht ihm sein neuer Partner meist gleich oder doch schon nach wenigen Minuten Platz. Setzt man an Stelle des alten Wächters eine fremde Assel an den Eingang, dann wird sie immer auf das heftigste abgewehrt.

Auch wenn der neue Partner nicht baut, kennt ihn der Wächter spätestens nach 24 Std. Markiert man beide individuell, dann zeigt sich, daß sie sich wenigstens 1- bis $2 \mathrm{mal}$ am Tag beim Wächterdienst ablösen. Hierbei genügt oft ein einziger Wechsel zum endgültigen Kennenlernen. Ursache für die geringe Zahl der notwendigen Ablösungen dürfte sein: Während der Wächter seinen Partner nur betasten kann, wenn dieser an ihm vorbei in die Höhle will, hat die nicht Wächterdienst versehende Assel, da sie normalerweise ebenfalls mit dem Kopf zum Höhleneingang sitzt, Gelegenheit, den Wächter dauernd mit den Antennen zu betasten. Die Voraussetzung für das Kennenlernen auf diese Weise, die Identität des Partners auch beim Fühlerberühren am Hinterende feststellen zu können, ist erfüllt: Hält man Asseln mit der Pinzette fest und nähert sie dem Wächter mit dem Hinterende voran, dann unterscheidet dieser seinen Partner von fremden Asseln, wie seine Verhaltensreaktionen - Platz machen bzw. Schlagen und sich Festklemmen - einwandfrei beweisen.

\section{Das Verhalten der verpaarten Asseln zueinander}

Zwischen den beiden Partnern herrscht Arbeitsteilung: Während der eine im Höhleninnern sitzt, die Höhle von Kot und hineingefallenen Fremdkörpern reinigt oder sich zur Nahrungssuche außerhalb der Höhle aufhält, verrichtet der andere Wächterdienst. Nicht in einem einzigen Fall konnten wir feststellen, daß auch nur für Minuten beide Asseln gleichzeitig die Höhle verlassen hätten. Beim Graben und Austragen von Kot konnten keine geschlechtsspezifischen Unterschiede in der Häufigkeit dieser Tätigkeiten festgestellt werden. Dagegen bestehen solche Unterschiede in der zeitlichen Verteilung des Wächterdienstes (im März und der ersten Aprilwoche, später konnten keine umfangreicheren Kontrollen mehr vorgenommen werden): Die ôt sind bevorzugt während der Aktivitätsphasen Wächter. So war am 13./14. und 15. 3. während der morgendlichen Aktivitätszeit in 77 von einem Pärchen bewohnten Höhlen in 63 das $\sigma^{\star}$ Wächter; während der mittäglichen Ruhezeit dagegen hielt in 58 von 79 Höhlen das $q$ Wache. $q 9$ halten sich im Frühjahr häufiger und länger zur Nahrungssuche außerhalb der Höhle auf, vermutlich deshalb, weil sie zur Eiproduktion mehr Nahrung benötigen. Es sind bevorzugt ơ, die - solange das Pärchen 
noch keine Jungen hat - Futter eintragen; von 53 im Laufe des März beim Futtereintragen gefangenen Asseln waren 39 우우.

Nur sehr sporadisch tragen einzelstehende Asseln Nahrung ein (vor allem vertrocknete, $z$. T. auch grüne Pflanzenteile, mit Flechten bewachsene Sandklümpchen und hin und wieder auch Ziegenkot). Häufiger sieht man diese Verhaltensweise bei verpaarten Asseln. Regelmäßig, d. h. täglich wenigstens einmal, tragen nur solche Asseln Futter ein, die Junge haben. Von sehr seltenen Ausnahmen abgesehen, nimmt die eintragende Assel zunächst selbst ausgiebig Nahrung zu sich: packt dann ein geeignetes Nahrungsobjekt mit den Mandibeln - oft helfen auch die beiden ersten Beinpaare noch beim Festhalten - und trägt es aus Entfernungen bis etwa $1 \mathrm{~m}$ zum Höhleneingang. Dort wird die Nahrung entweder direkt dem Wächter übergeben oder am Eingang deponiert und dann in den nächsten Minuten vom Wächter in die Höhle gezogen. In drei von fünf Fällen geht die eintragende Assel gleich anschließend wieder von der Höhle weg, nimmt erneut Nahrung zu sich und/oder trägt noch 1- bis 3mal Futter ein. Kleinere Objekte verzehrt der Wächter oft vollständig, von größeren nagt er nur Teile ab und schafft den Rest dann nach unten in die Höhle. (Auf das Futtereintragen wird an anderer Stelle näher eingegangen werden.)

\section{Das Verhalten nach Verlust des Partners}

H. reaumuri hat in den uns bekannten Biotopen kaum natürliche Feinde. Lediglich Walzenspinnen (verschiedene Arten der Familie Galeodidae), eine noch nicht bestimmte Araneen-Art und nach Gefangenschaftsbeobachtungen auch Skinke scheinen mit einiger Regelmäßigkeit Wüstenasseln zu verzehren. Die Ausfälle durch Feinde sind daher sehr gering. Größere Verluste treten durch weidende Ziegen und Schafe auf, die nahrungssuchende Asseln zertreten. Ein kleiner Prozentsatz von Asseln stirbt nach der Paarbildung eines nicht gewaltsamen Todes. Partnerverluste treten auch dadurch auf, daß einzelne Asseln, die bei der Nahrungssuche mehrfach erschreckt werden und deshalb längere Fluchtläufe unternehmen, ihre Höhlen trotz stundenlangen systematischen Suchens nicht mehr finden. Nichts deutet darauf hin, daß sich während der Beobachtungszeit Partner ,freiwillig“ trennen.

Wie reagiert eine Assel auf den Verlust ihres Partners, mit dem sie wenigstens einige Tage zusammengelebt hat? Versuche zu Beginn der Fortpflanzungsperiode erbrachten folgende Ergebnisse: Entfernt man den Partner vor oder gleich zu Beginn einer Aktivitätsphase, dann darf während dieser Phase keine fremde Assel in die Höhle, auch wenn sie sich noch so intensiv bemüht. Während der zweiten Aktivitätsphase lehnten noch 12 von 20 Asseln einen neuen Partner ab. Während der dritten ließen dagegen schon 15 von 20 Versuchstieren eine fremde, verschiedengeschlechtliche Assel ein. Nach 48stündiger Trennung von ihrem Partner waren es 19 von 20 Asseln, die eine fremde Assel als neuen Paarpartner akzeptierten. Die neu eingelassenen Asseln wurden nie mit dem verlorenen Partner verwechselt, sie wurden immer erst nach 1 bis $5 \mathrm{Std}$. dauernden Bemühungen endgültig eingelassen.

Setzte man - wenn sich die beiden neuen Partner schon kannten und vollkommen ungehindert gegenseitig in die Höhle ließen - am zweiten bis vierten Tag nach der Trennung den ehemaligen Partner der einen Assel (Experimente mit längerer Trennung wurden nicht durchgeführt) wieder an die Höhle, dann ließ diese, falls sie Wächterdienst versah, ihren alten Partner in den 4 geprüften Fällen gleich in die Höhle. Sie erkannte ihn also noch. Anschließend kam es immer zwischen den beiden gleichgeschlechtlichen Asseln $\mathrm{zu}$ Auseinandersetzungen, in denen $2 \mathrm{mal}$ der alte und $2 \mathrm{mal}$ der neue Partner Sieger blieb.

Versetzt man eine futtersuchende verpaarte Assel, dann sucht sie stundenlang nach ihrer Höhle: Fremden Höhlen nähert sie sich sehr zögernd; meist dreht sie schon kurz vor dem Eingang ab. Manchmal beugt sie sich auch ein kleines Stück in die Höhle hinein und berührt mit ihren Fühlerspitzen die 
Höhlenwand oder den Wächter. Auch wenn der Wächter nicht im geringsten reagiert, geht sie gleich wieder von der Höhle weg und sucht weiter. Frühestens am Ende der Aktivitätsphase, in der sie versetzt wurde, sehr oft aber auch erst während der nächsten Aktivitätsphase, beginnt die bei der Suche nach ihrer Höhle erfolglose Assel, sich für Höhlen mit fremden Asseln (oder auch leere fremde Höhlen) zu interessieren. Die in der Höhle zurückgebliebene Assel wartet also im Mittel etwas länger auf ihren Partner (s. o.), als dieser nach ihr sucht.

Nur mit fünf frisch gehäuteten $\varphi^{9} 9$ ) (Hinterende gehäutet) konnten Versuche gemacht werden. In den drei Fällen, in denen sie Wächter waren, wurden sie $2 \mathrm{mal}$ von einem Eindringling- $\hat{\alpha}$ aus der Höhle gezogen und umgebracht. (Sie können sich nicht richtig verklemmen; bei der geringsten Verletzung tritt Haemolymphe aus, was Artgenossen fast immer zu Kannibalismus anregt.) Am dritten $q$ quetschte sich ein $\sigma^{*}$ vorbei und warf es sofort und endguiltig aus der Höhle. Die zwei restlichen 오 wurden mehrfach an Höhlen verschiedener einzelstehender ${ }^{0} 0 \hat{}$ gesetzt. Sie bemühten sich - wohl durch die Weichheit der frisch gehäuteten Partien bedingt - nicht sehr intensiv und wurden in keinem Fall eingelassen. Nach 2- bis 3stündigem Aufenthalt außerhalb einer Höhle zeigten sich bereits Schrumpfungen und Verkrüppelungen an den frisch gehäuteten Paraeomeren und Peraeopoden. Die 우 waren danach nicht mehr in der Lage, ihren Vorderkörper vollständig zu häuten und starben.

In den folgenden Versuchen wurde untersucht, wie trächtige $q q^{10}$ ) auf den Verlust ihres Partners reagieren. Im ersten Experiment wurden aus 9 natürlichen von Pärchen mit trächtigen $q \uparrow$ bewohnten Höhlen die $\hat{o} \widehat{\jmath}$ entfernt. Im selben etwa $3 \times 10 \mathrm{~m}$ umfassenden Gebiet befanden sich außer den trächtigen 우 noch 5 von ihren Partnern getrennte, noch nicht gehäutete of우 allein in einer Höhle. Es wurde nun überprüft, ob und bei welchen 우오 es zu neuen Paarbildungen kam. Das Ergebnis (Tab. 5) ist vollkommen eindeutig. Es kann auch bei der geringen Zahl von Versuchen nicht auf Zufall beruhen, denn die Höhlen der nicht trächtigen 9 P lagen gleichmäßig verteilt zwischen denen der trächtigen. Partnersuchende Eindringlinge mußten daher fast doppelt so häufig auf Höhlen trächtiger fo stoßen.

Ein wesentliches Ergebnis dieses Versuchs ist noch, daß keines der alleinbleibenden trächtigen 우 innerhalb von 13 Tagen nach Trennung von seinem Partner aus der Höhle auswanderte. Nichtträchtige \&q wären, hätte sich kein Partner bei ihnen eingefunden, während dieser Zeit mit Sicherheit ausge-

Tab. 5: Gehen trächtige $q 0$ nach Verlust ihres Partners im natürlichen Lebensraum eine neue Paarbindung ein? Im selben Areal befanden sich auch 5 von ihren ${ }^{*} \sigma^{\star}$ getrennte nicht trächtige $\nmid \circ$ allein in einer Höhle (s. Text)

\begin{tabular}{|c|c|c|}
\hline \multirow{2}{*}{$\begin{array}{l}\text { Tage nach der } \\
\text { Trennung vom Partner }\end{array}$} & \multicolumn{2}{|c|}{ Zahl der neuen Paarbildungen mit } \\
\cline { 2 - 3 } & $5 q q$ nicht trächtig & 9 $q$ trächtig \\
\hline 2 & $1^{\mathrm{a}}$ & - \\
\hline 4 & $2^{\mathrm{a}}$ & - \\
\hline 13 & 5 & - \\
\hline
\end{tabular}

a Diese $\sigma^{\star} \sigma^{\star}$ wurden gleich wieder aus der Höhle entfernt.

9) Assel- 99 machen vor der Eiablage eine Parturialhäutung durch, bei der zuerst das Hinterende und dann mit artspezifisch verschiedenem Zeitabstand, bei $H$. reaumuri im Mittel nach $50 \mathrm{Std}$. ( $n=16$ Individuen), das Vorderende gehäutet wird (vgl. z. B. KaEstner 1959). Auf den Problemkreis: Paarbildung und Fortpflanzungszyklus wird an anderer Stelle eingegangen werden.

10) Trächtig heißt hier: die 우 tragen ihre Eier bereits in der Bruttasche. 
wandert und hätten sich auf aktive Partnersuche begeben. Das Resultat dieses Versuchs läßt nicht erkennen, wer - ob trächtiges $q$ oder Eindringling- $\sigma^{\star}$ oder beide - eine neuerliche Paarbildung verhindert. Folgende Experimente sollten diese Frage klären:

1. 24 trächtige 오 wurden aus ihren Bauten ausgegraben und - von ihrem Partner getrennt - in künstliche Höhlen gesetzt, die sie immer sofort bezogen. Je Gefäß mit 4 künstlichen Höhlen wurden $4 \delta^{*}{ }^{\hat{x}}$ zugesetzt, die ihrerseits schon mehr als 3 Tage von ihrem Partner isoliert worden waren und sich alle intensiv um einen neuen Partner bemühten. 3 Aktivitätsphasen nach dem Zusammensetzen hatte nur ein einziges der trächtigen 우우 ein $\sigma^{\star}$ eingelassen. Alle übrigen ${ }^{t} \delta$ hatten, obwohl sie sich nicht weniger intensiv als bei einem nichtträchtigen $q$ bemühten, keinen Einlaß erhalten.

2. Wieder wurden 4 우 je Gefäß - diesmal aber 2 trächtige und 2 nichtträchtige - in künstliche Höhlen gesetzt und sobald sie die Bauten bezogen hatten, 2 s zugesetzt. Von 27 Paaren, die sich während 3 Aktivitätsphasen bildeten, war in 26 Fällen das $q$ nicht trächtig. Direktbeobachtungen des Verhaltens zeigten auch bei diesem Versuch, daß sich die $\hat{o}^{*}{ }^{*}$ bei trächtigen 우 nicht weniger intensiv bemühten als bei nichtträchtigen. Sie wurden aber durch die sehr heftige Abwehr der trächtigen 우우 vertrieben.

3. Als Eindringlinge scheinen die $\delta^{\star} 0^{\wedge}$ nichtträchtige 우 von trächtigen nicht zu unterscheiden. Machen sie auch keinen Unterschied, wenn sie selbst Wächter und die 울 Eindringlinge sind ? o $\sigma^{*}{ }^{*}$, die wenigstens 3 Tage von ihren 우우 getrennt worden waren, wurden in künstliche Höhlen gesetzt. Zu jewreils 5 đơ in einem Gefäß wurden 4 우, 2 trächtige und 2 nichtträchtige gesetzt. 20 von insgesamt 24 워 wurden während der ersten Aktivitätsphase nach Versuchsbeginn eingelassen, und zwar 10 trächtige und 10 nichtträchtige. Von den trächtigen warfen 5 das $\hat{A}$ schon in der 1 . Aktivitätsphase aus der Höhle hinaus. Zwei weitere folgten ihrem Beispiel während der nächsten Aktivitätsphase. Bei nichtträchtigen ơ kam dies unter vergleichbaren Umständen nie vor.

Nach diesen Versuchsergebnissen sind es also allein die trächtigen 우, die eine erneute Paarbildung sehr erschweren und im natürlichen Biotop wohl weitgehend verhindern. ${ }^{11}$ )

Unter experimentellen Bedingungen im Labor kommt es noch regelmäßig zu Paarbildungen mit trächtigen 우: In einem relativ engen Gefä $\beta(10 \times 10 \mathrm{~cm}$ Grundfläche) befindet sich nur eine Höhle. $\mathrm{Zu}$ dem trächtigen \%, das die Höhle bereits 2-5 Tage bewohnt, wird ein đo gesetzt. Die Temperatur- und Feuchtigkeitsbedingungen erlauben diesem, auch einen Aufenthalt von 3 bis 5 Tagen außerhalb der Höhle schadlos zu überstehen. Innerhalb von $4 \mathrm{~T}$ agen hatte sich zwischen allen 40 trächtigen 우 (und 15 우, deren Junge zwischen 2-10 Tagen vor dem Versuchsbeginn ausgeschlüpft waren) und $\delta^{3} \sigma^{\star}$ neue Paare gebildet, die in den folgenden Wochen und Monaten ohne Störung ihres „Verhältnisses" bestehenblieben.

Von $\hat{\partial} \hat{o}$ isolierte 우, bei denen zwischen dem Ausschlüpfen der Jungen und einem dann erfolgenden Paarbildungsversuch $4-6$ Wochen vergangen waren, akzeptierten - mit oder ohne Jungtiere - unter den obengenannten Bedingungen auch bei 14tägigem Zusammenhalten kein fremdes ô mehr. Zu diesem Zeitpunkt (Mitte Juli) ließen aber auch ô $\widehat{o}$ keine fremden 우우 mehr zu sich in die Höhle. Paare, die sich früher bildeten, bleiben nach den bisherigen Laborbeobachtungen bis zum Tod eines Partners bzw. bis zur Auflösung des Familienverbandes im Frühjahr zusammen.

11) Anmerkung bei der Korrektur: Nach den Ergebnissen neuester Laborversuche scheinen

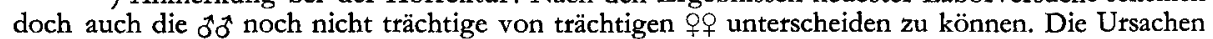
der Reaktionsunterschiede bei den Freiland- und den Laborversuchen sind noch nicht mit Sicherheit bekannt. 


\section{Diskussion}

Da oben ausschließlich Paarbildung und Paarzusammenhalt besprochen wurden, wollen wir hier nur diskutieren, inwieweit den Paarpartnern unmittelbare ökologische Vorteile aus dem monogamen Paarzusammenhalt erwachsen. Die Bedeutung der Monogamie beim Fortpflanzungsverhalten, der Brutpflege und der Entwicklung der Elternfamilie zum Familienverband werden an anderer Stelle behandelt werden.

In den Sommermonaten kann sich H. reaumuri in der Zeitspanne, in der die Witterungsverhältnisse eine Außenaktivität erlauben, keine Höhle von schutzbietender Tiefe in den ausgetrockneten, steinharten Boden graben. Sie ist darauf angewiesen, sich dann in eine schon vorhandene Höhle zurückziehen zu können.

Setzt man eine Wüstenassel, die sich in Höhlensuchstimmung befindet, in einem durchschnittlich dicht besiedelten Gebiet aus (2-3 Höhlen je $\mathrm{m}^{2}$ ), dann kann es trotz systematischem Suchen länger als eine Stunde dauern, bevor sie den ersten Höhleneingang findet. Würden Asseln am Ende der Aktivitätsphasen oder bei plötzlich einsetzenden ungünstigen Witterungsveränderungen „,auf gut Glück“" nach einem Höhlenplatz suchen, dann bestünde die Gefahr, $\mathrm{da} ß$ sie ihn nicht finden, entweder weil sie auf überhaupt keine oder nur auf schon besetzte Höhlen stoßen. Soll eine Assel mit tragbarem Zeitaufwand einen sicheren Höhlenplatz finden, dann muß sie sich in eine Höhle zurückziehen können, deren Lage ihr zumindest annähernd bekannt ist und die außerdem nicht überbesetzt sein darf.

Wann ist eine Höhle überbesetzt? Diese Frage kann nur dann richtig beurteilt werden, wenn man beachtet, daß: 1. die Jungtiere in den Höhlen aufwachsen und dort bis zum folgenden Frühjahr zusammenbleiben, 2. ein Weibchen bis zu 60 überlebende Junge produziert, 3. das Nahrungsangebot infolge der Hitze und Dürre zum Herbst hin schlechter wird, und 4. der Ernährungstaum infolge des kleinen Aktionsradius (1-2m) der Asseln, dessen Ursachen u. a. in der geringen Leistungsfähigkeit der Orientierungsmechanismen zu suchen sind, nur einer begrenzten Anzahl ausreichende Nahrung bieten kann. Bei Beachtung dieser Gesichtspunkte scheint es biologisch sinnvoll, wenn die Zahl der sich fortpflanzenden qo auf eines je Höhle beschränkt wird.

Die ausgeprägte Neigung der Asseln, nach Möglichkeit keine Höhle selbst $z u$ bauen, sondern schon vorhandene zu besetzen, dient zwar in idealer Weise dazu, den Asseln überflüssige Bauarbeit zu ersparen und bewirkt durch die ausgedehnten Suchgänge gleichzeitig eine Verbreitung der Art über den besiedelbaren Raum, läßt sich aber nicht ohne weiteres mit dem Bedürfnis in Einklang bringen, ihr einen sicheren Platz in einer bestimmten Höhle zu garantieren: Solange sie allein ist, kann sie die Höhle entweder nicht verlassen (vgl. das Verhalten von Eindringlingen, die eine leere Höhle gefunden haben, S. 136), was sich aber aus Ernährungsgründen auf die Dauer nicht durchführen läßt, oder aber sie läuft bei jedem noch so kurzzeitigen Ausflug Gefahr, ihren Bau beim Zurückkommen besetzt vorzufinden.

Die günstigste Lösung des gesamten Problemkomplexes ist die bei $H$. reaumuri verwirklichte pärchenweise Besiedlung einer Höhle: Die Zahl der qo+ wird auf eines je Höhle beschränkt. Da das Zahlenverhältnis von $o^{\star}{ }^{\star}$ und 9 ? etwa 1: 1 ist, hat jedes $q$ und jedes ot die Chance, einen Partner zu bekommen. Nur jeweils jede 2. Assel muß eine leere Höhle finden bzw. mit dem Bau einer neuen Höhle beginnen. Nach der Paarbildung können sich ot und o die Grabarbeit teilen. Vor allem aber kann immer ein Partner in der Höhle bleiben 
und diese verteidigen, während der andere auf Nahrungssuche geht. Diese bei $H$. reaumuri gesetzmäßige Regelung bringt ein großes Problem mit sich: Die in der Höhle als Wächter zurückbleibende Assel muß ihren Partner - wenn diesem der Höhlenplatz erhalten bleiben soll - von fremden Asseln beider Geschlechter unterscheiden können. Gegenüber ihrem Partner muß sie die gegenüber allen übrigen Artgenossen notwendige Aggressivität unterdrücken. Diesen Anforderungen wird eine auf Ortsbindung beruhende anonyme Monogamie, bei der Partner, die sich im gleichen Stadium des Fortpflanzungszyklus befinden, beliebig austauschbar sind (wie wir sie u. a. von verschiedenen Käferarten (z. B. Necrophorus: Pukowski 1933) und Decapoden (z. B. Hymenocera $\left.{ }^{12}\right)$ : WICKLER und SEIBT 1970) kennen) nur schwerlich gerecht: Würde der jeweilige Wächter nur gleichgeschlechtliche Artgenossen von der Höhle vertreiben, verschiedengeschlechtliche aber einlassen, dann wäre für eine verpaarte Wüstenassel das Risiko, die Höhle nach einem Ausflug besetzt vorzufinden, nur um 50\% vermindert. (Für eine frisch gehäutete Assel wäre es sogar innerhalb der Höhle lebensgefährlich, sie ist auf den Schutz ihres Paarpartners angewiesen, vgl. S. 149.) Trotz einer anonymen auf Ortsbindung basierenden Monogamie ließe sich diese Anforderung erfüllen, wenn sich das Verhalten des Partners an seiner Höhle und das eines fremden Eindringlings so unterschieden, daß der Wächter beide auseinanderhalten könnte. Dieser Weg ist bei der Evolution der Paarbindungsmechanismen von $H$. reaumuri nicht eingeschlagen worden. Es ist diejenige Lösung selektioniert worden, die wohl gegen Störungen weitaus am unempfindlichsten ist: das individuelle, nicht an einen bestimmten Ort und/oder bestimmte Verhaltensweisen gebundene Erkennen des Paarpartners.

Ein monogamer Paarzusammenhalt, der auf echtem individuellem Erkennen des Paarpartners beruht, wurde unseres Wissens außerhalb der Asselgattung Hemilepistus (nach MARIKovskys Beobachtungen erkennen sich auch bei $H$. rbinoceros die Partner eines Paares) noch bei keinem Wirbellosen nachgewiesen. Selbst unter Wirbeltieren sind - von Vögeln abgesehen - bislang nicht sehr viele derartige Fälle bekanntgeworden, bei Amphibien und Reptilien z. B. anscheinend noch keiner (vgl. die zusammenfassenden Darstellungen bei Erbl-Erbesfeld 1967 und WiCKLER 1967).

Da wir zu wenig vom Verhalten anderer Oniscoiden wissen, ist es derzeit noch sinnlos, zu diskutieren, über welche Zwischenstufen sich die Monogamie bei $H$. reaumuri vermutlich entwickelt hat. Die meisten Landasseln, die in unseren Breiten vorkommen, scheinen in offenen, anonymen Verbänden zu leben, in denen sich höchstens andeutungsweise subsoziale Phänomene nachweisen lassen (wie erhöhtes Wachstum innerhalb einer größeren Gruppe (Amouriq 1967); Zunahme der Aktivität innerhalb einer Gruppe bis zu einer oberen optimalen Grenze der Individuenzahl (MENER 1967)).

Abschließend ist festzustellen, daß sich der große Erfolg von $H$. reaumuri in ihrem Lebensraum nur begreifen läßt, wenn man die überragende Bedeutung ethologischer Anpassungen erkannt hat: Erst die Kombination des Grabverhaltens mit einem in manchen Aspekten hochentwickelten Sozialverhalten ${ }^{13}$ ) machen es verständlich, wie eine physiologisch mangelhaft angepaßte Assel zu den häufigsten Bewohnern eines während vieler Monate trocken-heißen Extrembiotops werden konnte.

12) Nach neueren Untersuchungen (WICKLER 1970 u. mündl. Mitt.) kennt bei Hymenocera das $\tau$ sein $q$ individuell, das $\%$ aber anscheinend nicht sein $\delta .-13$ ) Der Begriff Sozialverhalten wird hier nicht mit den bei Untersuchern sozialer Insekten üblichen Einschränkungen gebraucht. Er wird allgemeiner (vgl. z. B. Tinbergen 1955) definiert als: ein Komplex von Verhaltensweisen, bẹi dẹm zwẹei odẹr mẹhr Artgenossẹn zușammẹnaţbeiten, 


\section{Zusammenfassung}

1. Hemilepistus reaumuri lebt in nordafrikanischen und kleinasiatischen Steppen-, Halbwüsten-, stellenweise auch echten Wüstengebieten. Ihre mange]hafte physiologische Anpassung an die während vieler Monate trocken-heißen Biotope kompensiert die Wüstenassel durch ethologische Adaptationen so gut, daß sie - gemessen an der Individuendichte - vielerorts zu den erfolgreichsten Bewohnern der genannten Lebensräume zählt.

2. Voraussetzung für die im März, April erfolgende Paarbildung ist der Höhlenbesitz eines Partners ( $\delta$ oder o). Eine neue Höhle gräbt sich eine Wüstenassel nur dann, wenn sie auch bei tagelangem Suchen keine alte leere Höhle bzw. einen alleinstehenden höhlenbesitzenden Geschlechtspartner, der sie in seinen Bau läßt, findet.

3. Der einzelstehende Besitzer einer Höhle verteidigt diese zunächst gegen jeden Artgenossen. In einer im Mittel etwa 2 Std. dauernden Prozedur, läßt er einen einzelnen verschiedengeschlechtlichen Artgenossen in seine Höhle. Die Unterscheidung des gleich- vom verschiedengeschlechtlichen Artgenossen erfolgt höchstwahrscheinlich an Hand spezifischer chemischer Substanzen (Pheromone). Die Verhaltensweisen der Partner bei der Paarbildung sind nicht geschlechts-, sondern nur statusspezifisch (= eine Assel ist unabhängig von ihrem Geschlecht entweder der ,Wächter“ oder der „Eindringling“). Versucht tagelang kein Artgenosse in die Höhle einer einzelstehenden, verpaarungsbereiten Assel einzudringen, dann verläßt sie die eigene Höhle und wird zum partnersuchenden Eindringling.!

4. Spätestens 24 Std. nach dem ersten Kontakt am Höhleneingang kennen sich die Partner eines Paares individuell. Sie lassen sich dann gegenseitig ohne Behinderung in die Höhle, wogegen sie jede fremde Assel unabhängig von ihrem Geschlecht abwehren. Das individuelle Erkennen ist nicht an die eigene Höhle oder an bestimmte Verhaltensreaktionen gebunden. Ausschaltversuche zeigen, daß die individuellen Merkmale beim Betasten mit dem Endglied der 2. Antenne wahrgenommen werden. Es handelt sich vermutlich um chemische Merkmale. Partner, die man getrennt hat, erkennen sich auch nach mehreren Tagen noch einwandfrei.

5. Zwischen den Paarpartnern kommt es beim Bau, der Reinigung und Verteidigung der Höhle, bei der Nahrungsbeschaffung (und später im Jahr auch bei der Brutpflege) zu Arbeitsteilung.

6. Eine „freiwillige“ Trennung von Paarpartnern im Jahr der Paarbildung konnte nicht beobachtet werden. Verpaarte $q q$ gehen nach Verlust ihres $\delta$ eine neue Paarbindung ein, solange sie noch nicht trächtig sind. Trächtige ơ und $q$ +, die bereits Junge haben, scheinen sich im Freiland nach einem Partnerverlust nicht mehr zu verpaaren.

7. Das Zusammenleben der Wüstenasseln in monogamen Paaren, deren Zusammenhalt durch die individuelle Bindung der Partner aneinander (und daneben natürlich auch durch ihre gemeinsame Bindung an die Höhle) gesichert wird, bringt in dem bewohnten Lebensraum große Selektionsvorteile mit sich. Es verhindert die Überbesetzung einer Höhle, die sich die Asseln der Beschränktheit ihres Aktionsradius und der Knappheit der Nahrung wegen nicht leisten können, und dient damit gleichzeitig der Verbreitung der Art über den besiedelbaren Raum. Es erspart den Asseln den Bau nicht unbedingt notwendiger Höhlen und garantiert jeder Assel einen lebensnotwendigen Höhlenplatz während der heißen Jahreszeit. 


\section{Summary}

\section{Pair formation and pair maintenance in the monogamous desert wood louse Hemilepistus reaumuri}

1. Hemilepistus reaumuri lives in North African und Arabian steppes, semideserts and, in some areas, real desert. Their poor physiological adaptation to the dry and hot conditions prevalent for many months of the year is so well compensated for by ethological adaptations that the woodlice (as measured from population density) are some of the most successful inhabitants of the above mentioned regions.

2. The precondition for pair formation, which takes place in March and April, is the possession of a burrow by a prospective partner (be it $\hat{o}$ or $q$ ). A new burrow is dug only when a woodlouse, after days of searching, cannot find an old and empty burrow or a burrow which contains a prospective mate.

3. Initially the single owner of a burrow defends its home against every intraspecific intruder. After a ritual which lasts for about two hours, the owner will allow entry of an individual of the opposite sex. Sex difference is most probably communicated by a specific chemical substance (pheromone). The behaviour pattern of the prospective partners during pair formation is not a sexually specific but a status specific function (i. e. the defensive or intrusive behaviour of a woodlouse does not depend on its sex). If no woodlouse attempts entry of an occupied burrow of a single sexually mature animal then the occupant will leave its home and adopt the role of the intruder.

4. 24 hours (maximal) after their first contact at the mouth of a burrow a pair of woodlice exhibit mutual recognition of one another. One will allow the other to re-enter the burrow without hindrance whereas a stranger will be driven off. Individual recognition is not bound to the own burrow, nor is it released by a particular behaviour pattern by the partner. As ablation experiments have shown, recognition of the individual is probably released by specific chemical substances (pheromones), perceived by receptors situated on the tips of the second antennae. Pairs of animals which have been forcibly parted still recognize one another without any difficulty after several days separation.

5. There is a division of labour between the two members of an established pair in building, cleaning and defense of the burrow, in food collection and later in the year in the care of the young.

6. Our observations could not establish that "voluntary divorce" of a pair ever took place in the year of pair formation. Paired females accepted a new male if their previous partner perished and if they were not already pregnant. Pregnant females and females with young did not, under natural conditions, accept a new partner.

7. The monogamy of desert woodlice, which is maintained by mutual fixation on the partner (and also by their fixation on the burrow) is of great selective advantage in such harsh environmental conditions. Monogamy prevents over-population of a burrow, which would be disasterous considering the small foraging range and the paucity of available food. On the other hand it cares for the distribution of the species over the territory suitable for colonization. It allows great economy, with respect to the construction of new burrows, and also guarantees that every woodlouse has a life-preserving shelter during the hot part of the year. 


\section{Literaturverzeichnis}

Amovriq, L. (1967): Rôle des effets de groupe et de masse sur la croissance, la maturité sexuelle, la fécondité de jeunes de Porcellio scaber Latr. (Oniscidae, Isopoda). Bull. soc. zool. France 92, 177-185 - Cloudsley-Thompson, J. L. (1956a): Studies in diurnal rhythms. VI. Bioclimatic observations in Tunesia and their significance in relation to the physiology of the fauna, especially woodlice, centipedes, scorpions and beetles. Ann. Mag. Nat. Hist. (12), 9, 305-329 - Ders. (1956b): Studies in diurnal thythms. VII. Humidity responses and nocturnal activity in woodlice (Isopoda). J. Exp. Biol. 33, 576-582 - Edney, E. B. (1958): The microclimate in which woodlice live. Proc. Xth Intern. Congr. Entomol. 2, 709-712 • EIBL-ElBESFELDT, I. (1967): Grundriß der vergleichenden Verhaltensforschung. R. Piper u. Co. Verlag, München $\bullet$ FischвACH, E. (1954): Licht-, Schwereund Geruchssinn bei Isopoden. Zool. Jb. Physiol. 65, 141-170 • KAEstNER, A. (1959): Lehrbuch det Speziellen Zoologie. Teil I: Wirbellose, 4. Lieferung, 926-961. Gustav Fischer, Stuttgart Legrand, J. (1958): Comportement sexuel et modalités de la fécondation chez l'oniscoide Porcellio dilatatus Brandt. C. r. Acad. Paris Sci. 246, 3120-3122 • Marrkovsky, P. J. (1969): A contribution to the biology of Hemilepistus rhinoceros. Zool. Z. 48, 677-685, russisch mit engl. Zusammenfassung $\bullet$ MENER, B. (1967): Mise en évidence de l'effet de groupe et du phénomene subsocial chez Porcellio scaber Latr. (Oniscidae, Isopodes terrestres). Rev. Comp. Annal. 5, 37-44 - Mensching, H. (1968): Tunesien. Wissenschaftliche Buchgesellschaft, Darmstadt • Pukowski, E. (1933): Okologische Untersuchungen an Necrophorus F. Z. Morph. Okol. Tiere 27, 518-586 - TiNBergeN, N. (1955): Tiere untereinander. Paul Parey, Berlin u. Hamburg • VANDEL, A. (1924): Un cloporte qui se creuse un terrier. Bull. soc. zool. France 49, 469-471 • VERRIER, M. L. (1932): Etude des rapports de la forme, de l'habitat et du comportement de quelques Crustaces Isopodes. Bull. biol. France et Belg. 66, 200-231 - WARBURG, M. R. (1968): Behavioral adaptations of terrestrial isopods. Am. Zoologist 8, 545-559 - WICKLER, W. (1967): Vergleichende Verhaltensforschung und Phylogenetik. In: Heberer, G. (ed.): Die Evolution der Organismen. I, 420-508, 3. Aufl., Gustav Fischer, Stuttgart Ders. (1970): Harlekin-Garnele und Acanthaster-Problem (Monogamie-Studien). Naturwiss. Rdsch. 23, 368-369 - WICkLER, W., u. SEIBT, U. (1970): Das Verhalten von Hymenocera picta Dana, einer Seesterne fressenden Garnele (Decapoda, Natantia, Gnathophyllidae). Z, Tierpsychol. 27, $352-368$.

Anschrift der Verfasser: Dr. K. Eduard Linsenmair, Dr. Christa Linsenmair, Universität Regensburg, Lehrstuhl für Biologie II, D-8400 Regensburg 